\title{
Effective clustering for single cell sequencing cancer data
}

\author{
Simone Ciccolella ${ }^{\dagger}$, Murray Patterson ${ }^{\dagger, *}$, Paola Bonizzoni and Gianluca Della Vedova \\ Experimental Algorithmics Lab (algolab.eu), Dipartimento di Informatica, Sistemistica e \\ Comunicazione (DISCo), Università degli Studi di Milano-Bicocca, Milan, Italy \\ $\dagger$ Joint first author \\ * Corresponding author: murray.patterson@unimib.it
}

\begin{abstract}
Background. Single cell sequencing (SCS) technologies provide a level of resolution that makes it indispensable for inferring from a sequenced tumor, evolutionary trees or phylogenies representing an accumulation of cancerous mutations. A drawback of SCS is elevated false negative and missing value rates, resulting in a large space of possible solutions, which in turn makes infeasible using some approaches and tools. While this has not inhibited the development of methods for inferring phylogenies from SCS data, the continuing increase in size and resolution of these data begin to put a strain on such methods.

One possible solution is to reduce the size of an SCS instance - usually represented as a matrix of presence, absence and missing values of the mutations found in the different sequenced cells and infer the tree from this reduced-size instance. Previous approaches have used $k$-means to this end, clustering groups of mutations and/or cells, and using these means as the reduced instance. Such an approach typically uses the Euclidean distance for computing means. However, since the values in these matrices are of a categorical nature (having the three categories: present, absent and missing), we explore techniques for clustering categorical data - commonly used in data mining and machine learning - to SCS data, with this goal in mind.
\end{abstract}

Results. In this work, we present a new clustering procedure aimed at clustering categorical vector, or matrix data - here representing SCS instances, called celluloid. We demonstrate that celluloid clusters mutations with high precision: never pairing too many mutations that are unrelated in the ground truth, but also obtains accurate results in terms of the phylogeny inferred downstream from the reduced instance produced by this method.

Finally, we demonstrate the usefulness of a clustering step by applying the entire pipeline (clustering + inference method) to a real dataset, showing a significant reduction in the runtime, raising considerably the upper bound on the size of SCS instances which can be solved in practice.

Availability. Our approach, celluloid: clustering single cell sequencing data around centroids is available at https://github.com/AlgoLab/celluloid/ under an MIT license.

Keywords. clustering, single sell sequencing, cancer progression 


\section{Background}

A tumor, at the time of detection, usually by performing a biopsy on the extracted tissue, is the result of a tumultuous evolutionary process, originating from a single tumor cell - the founder cell [24] - that has acquired a driver mutation, which inhibits control on the proliferation of subsequent cancer cells. From that moment, the combination of unrestrained proliferation and a very hostile environment - as the immune system fights for survival, and so the tumor cells, under extreme selection pressure, have to disguise themselves to avoid being attacked, compete with each other, all while having to thrive while getting low levels of oxygen - produces the accumulation of highly elevated number of mutations, including structural variations. This model of tumor evolution is called the clonal model [24], since a clone is a population of cells carrying the same set of mutations. Understanding this clonal evolutionary history can help clinicians understand the cell heterogeneity in the tumors of various types of cancer and, more importantly, gives insights on how to devise therapeutic strategies [23, 31].

The rise of next-generation sequencing (NGS) technologies has led to the computational problem of tumor phylogeny inference from NGS bulk sequencing data [7, 21, 28, 11]. This idea is very costeffective, since NGS data is cheap to obtain and very reliable. The procedure consists of extracting different samples of the tumor and aligning the NGS reads against the reference genome: this allows to determine the approximate fraction of reads from each sample that are affected by any given mutation. This fraction is taken as a proxy of the fraction of cells in each sample that are affected by that mutation. The main difficulties of this technique are the fact that a sample contains a mix of both healthy cells and cancer cells, while the cancer cells are an unknown mixture of different clones.

Since both the composition of the samples and the evolutionary history are unknown in this model, most of the approaches have needed some simplifying assumption, such as the infinite sites assumption (ISA) which postulates that each mutation is acquired exactly once, and never lost, in the entire tree. While this assumption has only limited biological validity [20, 2] it reduces greatly the space of all possible solutions, making feasible several approaches [7, 11], which are at least partially inspired by a classical linear time algorithm [10] for reconstructing the phylogeny from noise-free character data: this latter computational problem is called the perfect phylogeny problem.

The newer single cell sequencing (SCS) technology provides a much finer level of resolution: in fact we can determine whether or not a given cell has a mutation, therefore avoiding the notion of sample and the approximations implied by the use of samples. Still, SCS is expensive and plagued by high dropout, i.e., missing values, and false negative rates.

Nowadays, we are witnessing a decrease in SCS costs, coupled with improvements in the dropout and false negative rates, stimulating the research on tools for tumor evolution inference from SCS data [16, 26, 4, 5, 32, 6]. We believe that this line of research is going to become even more important in the next few years, since currently available SCS data is associated with a very large solution space. Moreover, the high missing value and false negative rates allow a huge number of possible phylogenies with near optimal values of the objective function: this fact makes difficult to determine which methods actually produce better solutions and shows that the objective function is not able to fully capture the biological soundness of the phylogeny.

These advances in costs and quality of the data produced will result in larger, but more constrained, instances: the net effect is a considerable reduction in the number of likely solutions. Since most of the currently available methods do not scale well to large instances (usually their running time is quadratic with respect to the number of mutations), a preprocessing step can be useful to reduce the size of an instance: for example, SPhyR [6] uses $k$-means [22, 1] to such purpose. 
However, $k$-means is designed for continuous data - where means are usually based on a Euclidean distance - while SCS data, specifying the presence (1) or absence (0) of a mutation in a cell, or the fact that it is missing (2), can be thought of as categorical.

Clustering categorical data is an active field of research in data mining [18, where massive databases of categorical data are handled, e.g., finding groups of members of an insurance policy who regularly travel overseas.

In this paper we devise a new method for clustering categorical data, called celluloid. Not only does celluloid cluster categorical data (based on a $k$-modes framework), but because of its novel conflict dissimilarity measure — tailored to properties specific to SCS data (see Methods) — it obtains the best results in a comparison of a variety of different clustering approaches on SCS data. This comparison has three goals: (1) to assess which clustering methods are more precise on SCS data by comparing the actual clustering produced, (2) to evaluate the effect of a clustering step on the downstream phylogeny inference step, and (3) to assess the usefulness of a clustering step on real data, by showing its capability to reduce an instance that is too large for some of the current methods so that the clustered instance can be easily solved.

\section{Methods}

Starting with the method we devise, celluloid, we now give an overview of the methods we consider for clustering mutations (columns) of single-cell sequencing (SCS) datasets. We chose the clustering methods for this comparison based on: (a) the method clusters vector or matrix data into a smaller such set of vectors or matrix, as is the nature of SCS data; and (b) the method has an implementation available.

\section{Celluloid}

Our method celluloid is based on the $k$-modes framework [13, 14] which, given $m$ objects $X$ on $n$ categorical attributes $A=\left\{\bar{a}_{1}, \ldots, \bar{a}_{n}\right\}$ and an integer $k$, finds $k$ modes $Q=\left\{\bar{q}_{1}, \ldots, \bar{q}_{k}\right\}$ and an $m \times k$ partition matrix [12] that minimize the following objective function:

$$
\sum_{l=1}^{k} \sum_{j=1}^{m} \sum_{i=1}^{n} p_{j l} \delta\left(x_{j i}, q_{l i}\right)
$$

subject to

$$
\begin{aligned}
& \sum_{l=1}^{k} p_{j l}=1, \quad 1 \leq j \leq m, \\
& p_{j l} \in\{0,1\}, \quad 1 \leq j \leq m, 1 \leq l \leq k .
\end{aligned}
$$

That is, for each $\bar{q}_{l} \in Q, \bar{q}_{l i}$ is one of the $\bar{a}_{i}$ possible categories at position $i$. Note that every $\bar{a}_{i}=\{0,1,2\}$ when considering single cell sequencing data for inferring tumor evolution.

Here $d_{1}$ is a dissimilarity measure between object $\bar{x}_{j}$ and (mode) $\bar{q}_{l}$ :

$$
d_{1}\left(\bar{x}_{j}, \bar{q}_{l}\right)=\sum_{i=1}^{n} \delta\left(\bar{x}_{j i}, \bar{q}_{l i}\right),
$$

according to some dissimilarity $\delta$. A typical dissimilarity is the simple matching dissimilarity between categories: 


$$
\delta_{M}\left(\bar{x}_{j i}, \bar{q}_{l i}\right)=\left\{\begin{array}{ll}
0 & \text { if } \bar{x}_{j i}=\bar{q}_{l i} \\
1 & \text { o.w. }\left(\bar{x}_{j i} \neq \bar{q}_{l i}\right)
\end{array} .\right.
$$

Here, we design a new dissimilarity, based on the notion of conflict, to be used within the $k$ modes framework. Since a 2 represents missing data in our context, we are only interested in cases where a 2 is not involved, slightly relaxing the above matching dissimilarity of Equation 4 , obtaining what we call the conflict dissimilarity, formally described in Eq. 5 and used as a dissimilarity in the $k$-modes framework.

$$
\delta_{C}\left(\bar{x}_{j i}, \bar{q}_{l i}\right)= \begin{cases}0 & \text { if } \bar{x}_{j i}=2 \text { or } \bar{q}_{l i}=2 \\ \delta_{M}\left(\bar{x}_{j i}, \bar{q}_{l i}\right) & \text { o.w. }\end{cases}
$$

Now, a mode of cluster $C_{l} \subseteq X$ is a vector $\bar{q}_{l}$ which minimizes:

$$
D\left(C_{l}, \bar{q}_{l}\right)=\sum_{\bar{x}_{j} \in C_{l}} d_{1}\left(\bar{x}_{j}, \bar{q}_{l}\right) .
$$

Note that $\bar{q}_{l}$ is not necessarily an element of $C_{l}$. The $k$-modes algorithm then starts with some initial set $Q^{0}$ of $k$ modes, and an initial collection $\mathbb{C}^{0}$ of $k$ disjoint subsets of $X$, and then iterates the operations:

1. compute $d_{1}\left(\bar{x}_{j}, \bar{q}_{l}\right)$, where $\bar{q}_{l}$ is a mode of cluster $C_{l}^{0} \in \mathbb{C}$, for each $\bar{x}_{j}, C_{l}^{0}$;

2. allocate $\bar{x}_{j}$ to a cluster $C_{l^{\prime}}^{1}$ minimizing $d_{1}\left(\bar{x}_{j}, q_{l^{\prime}}\right)$; and

3. recompute a new set of modes $Q^{1}$ according to the new clusters $\mathbb{C}^{1}$, minimizing Equation 1 . subject to the constraints in Equation 2,

until convergence, i.e., until $Q^{t+1}=Q^{t}$. The modes in the third step above are found according to the following theorem, where $c_{r i}$ is the $r$-th category of attribute $\bar{a}_{i}$ and $f\left(\bar{a}_{i}=c_{r i} \mid X\right)$ is its relative frequency in $X$ :

Theorem 1 ([14]) Eq. [6 is minimized iff $f\left(\bar{a}_{i}=q_{l i} \mid X\right) \geq f\left(\bar{a}_{i}=c_{r i} \mid X\right)$ for $q_{l i} \neq c_{r i} \forall i \in[n]$.

In other words, Eq. 6 is minimized by selecting the mode category for each attribute. Note that this theorem implies that the mode of $X$ is not necessarily unique.

Since the solution depends on the initial set $Q^{0}$ of $k$ modes, we consider two procedures for initializing $Q^{0}$. The first one is quite simple: a random selection of $k$ objects from the set $X$ of objects as the initial $k$ modes - which we refer to as the random initialization procedure. The second one, devised in [14], is a more complicated procedure, based on the frequencies $f\left(\bar{a}_{i}=c_{r i} \mid X\right)$ of all categories, which we refer to as the Huang initialization procedure. We focus on this second procedure, since it achieves the best results:

1. order the categories of each attribute $\bar{a}_{i}$ in descending order of frequency, i.e. $f\left(c_{r_{1} i}\right) \geq$ $f\left(c_{r_{2} i}\right) \geq f\left(c_{r_{3} i}\right)$, etc.;

2. assign uniformly at random the most frequent categories to the initial $k$ modes;

3. for each $\bar{q}_{l}$, select the $\bar{x}_{j} \in X$ most similar to $\bar{q}_{l}$ and make this the mode $\bar{q}_{l}^{0} \in Q^{0}$, such that $\bar{q}_{l}^{0} \neq \bar{q}_{l^{\prime}}^{0}$ for $l \neq l^{\prime}$. 
This final step is to avoid empty clusters in $\mathbb{C}^{0}$. This initial selection procedure is to have a diverse set of initial modes $Q^{0}$, which can lead to better clustering results — see more details in [14.

Our approach, celluloid: clustering single cell sequencing data around centroids, is our conflict dissimilarity measure and the Huang initialization procedure, used within the $k$-modes framework its implementation available at https://github.com/AlgoLab/celluloid/ under an MIT license.

\section{$k$-modes}

Since there is package for computing a clustering based on the $k$-modes framework available on the Python Package Index (pypi) at https://pypi.org/project/kmodes/, we decided to use this in our tests as well. The above package comes with a variety of standard dissimilarity measures, as well as the Huang (and random) initialization procedures (see above).

Here we use the above implementation with the options of matching dissimilarity (Equation 4) and the Huang initialization procedure, since this combination of options produced the best results. This is what we refer to as $k$-modes in our experiments.

\section{$k$-means}

Given $m$ objects $X=\left\{\bar{x}_{1}, \ldots, \bar{x}_{m}\right\}$ on $n$ real values, i.e., each $x_{i} \in \mathbb{R}^{n}$ and an integer $k$, the $k$-means algorithm [22, 1] finds the vector of $k$ values, called means, $Q=\left\{\bar{q}_{1}, \ldots, \bar{q}_{k}\right\}$ and an $m \times k$ partition matrix minimizing the following objective function:

$$
\sum_{l=1}^{k} \sum_{j=1}^{m} p_{j l} d\left(\bar{x}_{j}, \bar{q}_{l}\right),
$$

subject again to the constraints in Equation 2 .

Here, $d$ is a distance measure that is usually the Euclidean distance, i.e.,

$$
d\left(\bar{x}_{j}, \bar{q}_{l}\right)=\sum_{i=1}^{n}\left(\bar{x}_{j i}-\bar{q}_{l i}\right)^{2} .
$$

The $k$-means algorithm starts with some initial set $Q^{0}$ of $k$ means, and an initial collection $\mathbb{C}^{0}$ of $k$ disjoint subsets of $X$, and then iterates the operations 1-3 as in the $k$-modes algorithm, but using the Euclidean distance (Equation 8 instead of dissimilarity, and computing means instead of modes, that minimize instead the objective function of Equation 7 , subject to the constraints in Equation 2.

In our experiments, we used the implementation of $k$-means clustering available at https: //scikit-learn.org/stable/modules/clustering.html\#k-means.

\section{Affinity propagation}

The affinity propagation algorithm [9] uses as input a set of similarities between data points. Those similarities are clustered by choosing a point as a representative for each class. Such points gradually emerge iteratively using a message-passing procedure, where each point exchanges messages to all other points.

In particular, there exist two types of messages: (1) responsibility $r(i, k)$ is sent from point $i$ to the candidate representative point $k$ reflecting the cumulative evidence of how well-suited $k$ is to serve as representative of $i$; and (2) availability $a(i, k)$ sent from the candidate representative 
$k$ to $i$ reflecting the cumulative evidence of how well-suited $k$ should be chosen by $i$ to be its representative. Both variables take into account other potential candidates.

Such messages are exchanged iteratively, each time refining $r(\cdot, \cdot)$ and $a(\cdot, \cdot)$, until a stop condition is fulfilled. At any point these variables can be combined to identify the representatives. For each point $i$, the value of $k$ that maximizes $r(i, k)+a(i, k)$ identifies the representative $k$ of $i$, where $k$ and $i$ can be the same point.

In our experiments, we used the implementation of affinity propagation clustering available at https://scikit-learn.org/stable/modules/clustering.html\#affinity-propagation.

\section{Hierarchical agglomerative clustering}

The hierarchical agglomerative clustering method [17] produces, in an hierarchical fashion, groups of disjoint subsets of a given set, each maximizing an internal similarity score. The procedure is executed in a bottom-up fashion in which, initially, each point is in a subset by itself. At each step, two sets are merged together, so that the union maximizes a given criterion. The procedure is then repeated until only one group remains, thus having the complete hierarchical structure of the clustering.

In our experiments we used the Manhattan metric, i.e., the sum of the horizontal and vertical distance of two points, to compute the similarity scores, as this metric is more suited for a matrix containing categorical data, as opposed to the Euclidean distance more suited for a continuousvalued matrix. The similarity of two sets of observations is computed using the average of the distances between elements in the respective sets. Consequently, as expected, the hierarchical agglomerative clustering, when using the Manhattan metric performed better (see Results) than the Euclidean metric, and so we only included the former in the comparison.

In our experiments, we used the implementation of hierarchical clustering available at https: //scikit-learn.org/stable/modules/clustering.html\#hierarchical-clustering.

\section{BIRCH clustering}

The BIRCH clustering procedure [33] takes as input a set of points as well as the desired number of clusters, and operates in four steps. (1) In the first step it computes the clustering feature (CF) tree while computing measures using a predefined metric. (2) The second optional phase tries to build a smaller CF tree while removing outliers and regrouping crowded subclusters into larger ones. (3) In the third step, a hierarchical clustering algorithm, usually an adaptation of the agglomerative clustering (briefly described in the previous subsection), is used to cluster all the leaves of the CF tree. During this phase, the centroids of each cluster are computed. (4) The centroids are then used for the final fourth step to further refine the clusters, since minor and localized misplacements could occur during the previous step. This last step can also be used to label the points with the cluster they are placed in - we used this feature to obtain the groups of mutations in our experiments.

In our experiments, we used the implementation of BIRCH clustering available at https:// scikit-learn.org/stable/modules/clustering.html\#birch.

\section{Spectral clustering}

The spectral clustering algorithm [27] - see [30] for a gentle and comprehensive treatment of the topic - first performs dimensionality reduction on the data, and then clusters, using a standard clustering technique such as $k$-means, the data in this reduced dimension. In order to reduce the dimensionality, it first constructs a similarity graph from the initial matrix of similarities of the 
input set of data points - usually a sparse representation of this similarity matrix, e.g., the $k$ nearest neighbor graph [30]. It then takes the Laplacian matrix [3] on this graph, and a subset of the (relevant) eigenvectors (spectrum) of this Laplacian matrix - itself a matrix, is the reduceddimension data. It is then this matrix which is then passed to the clustering technique.

In our experiments, we used the implementation of spectral clustering available at https: //scikit-learn.org/stable/modules/clustering.html\#spectral-clustering.

\section{Results}

To evaluate the accuracy of the clustering methods, we designed a two-fold experimentation on synthetic datasets: we first measure the quality of the clusters found by the methods, and later we evaluate how such clusters impact the quality of the phylogenies returned by a cancer progression inference method.

The simulated data are generated as follows. First we simulate a random tree topology on $s$ nodes, each representing a tumor clone, by first creating a root (the germline) and then iteratively attaching the $s-1$ remaining nodes uniformly at random to any other node in the tree. The nodes are then randomly labeled with $m$ mutations - meaning that each mutation is acquired at the node that it labels. Then, a total of $n$ cells are associated to the nodes, uniformly at random. A binary matrix $M$ is then extracted from these cells giving rise to a genotype profile for each cell (a row in $M$ ), which is the presence (1) or absence (0) of each mutation (column in $M$ ) in the cell, given the presence or absence of the mutations on the path in the tree from the root to this cell. The binary matrix is then perturbed according to the false negative, false positive and missing value rates, to simulate a real-case scenario. Each of the $s$ nodes is therefore considered as a natural (true) cluster of the simulated dataset.

For each experiment, we consider 100, 200 and 300 cells, respectively experiments 1,2 and 3 . For each such value we generated 50 simulated datasets, where we fixed the number $s$ of clones to 20 and the number $m$ of mutations to 1000 . While this number of mutations is at the high end in terms of currently available real cases, it will be a typical size in the near future - some such cases already existing today (see Application on read data).

We performed clustering on the datasets of our experiments to obtain instances with a reduced number of columns (mutations), which can in turn be given as input to such a cancer progression inference method above. The clustering methods we used were all the ones described in the Methods section, i.e., celluloid, $k$-modes, $k$-means, affinity propagation, hierarchical agglomerative, BIRCH and spectral clustering. Since the cancer inference methods tend to scale quadratically with the number of mutations, we produced 100 clusters with each clustering method, which is a reasonable number of mutations given the currently available literature.

\section{Evaluating a clustering}

To evaluate to the clusters obtained, we used standard precision and recall measures, adapted to the particular goal, as follows.

Precision: measures how well mutations are clustered together. For each pair of mutations appearing in the same clone in the simulated tree, we check if they are in the same cluster, resulting in a true positive $(T P)$. For each pair of mutations clustered together that are not in the same clone, we encounter a false positive $(F P)$. The value of the precision is then calculated with the standard formula: $\frac{T P}{T P+F P}$. 
Recall: measures how well mutations are separated. For each pair of mutations in the same clone, we now also check if they are not in the same cluster, resulting in a false negative $(F N)$. The recall is then calculated as: $\frac{T P}{T P+F N}$.

It is important to highlight that we are mostly interested in obtaining a high precision since, while cancer phylogeny inference algorithms can later cluster together mutations by assigning them to same subtree or the same path, they cannot separate mutations that have been erroneously clustered together.

In Figures 1, 2 and 3 - representing the respective experiments 1, 2 and 3 - a common trend is evident: indeed standard clustering methods ( $k$-means, affinity propagation, hierarchical agglomerative, BIRCH and spectral clustering) perform much more poorly than celluloid and $k$ modes, presenting a gap from all the other methods in terms of both precision and recall. On the other hand, celluloid and $k$-modes differ slightly in terms of precision.

It is interesting to notice that the precision of the conflict dissimilarity rapidly increases when the amount of cells increase, thus being well-suited for future increases on the size of SCS datasets.

We have also evaluated the quality of the clusters with some standard clustering metrics such as Adjusted Rand index [15], Fowlkes-Mallows index [8], Completeness score [25] and V-Measure [25]. In this case, we measure how much the computed clustering is similar to the ground truth.

In Figures 4, 5 and 6 - representing again, respectively, the results of experiments 1, 2 and 3 - we see the similar trend of celluloid and $k$-modes performing much better in terms of precision than all the other methods, presenting this same gap from all the other methods, while differing slightly from each other in terms of precision.

We decided also to compute Adjusted Rand index, Fowlkes-Mallows index, Completeness score and V-Measure for all pairs of techniques to allow observation of the similarity, according to the measures, between different algorithms. The heatmaps in figures 7,8 and 9 - representing, respectively, experiments 1,2 and 3 - show the average value of the scores of each simulated dataset for each pair of clustering method.

The first column of all the heatmaps represents the comparison with the ground truth, where in all the cases celluloid achieves larger values, thus more resembling the ground truth. Furthermore, we compare all pairs of clustering algorithms, to detect similarities between them; as expected, celluloid and $k$-modes are very similar to each other, while the other methods tend to be quite dissimilar to each other.

\section{Assessing the impact of a clustering}

To better understand the impact of the clustering on the actual cancer progression inference, we considered SCITE [16] and SPhyR [6], two published, publicly available inference tools tailored to SCS data, and have been shown to scale to instances of the size we consider in our study - the latter tool has a built-in clustering preprocessing step which uses $k$-means. We executed SCITE [16] and SPhyR [6] on both the obtained clusters and on the unclustered datasets, to understand the effect of the clustering on the tools. Furthermore we selected our own cancer progression inference tool SASC [4] that is not able to complete in reasonable time on the unclustered data, due to the higher complexity of the search space of the solutions it generates. We performed the inference with all the clustering methods used as a preprocessing step.

For assessing the accuracy of the methods we used the measures defined in [5, 4]:

Ancestor-Descendant accuracy: for each pair of mutations in an ancestor-descendant relationship in the ground truth, we check whether the relationship is conserved in the inferred tree 
$(T P)$ or whether it is not $(F N)$. For each pair of mutations in an ancestor-descendant relationship in the inferred tree, we also check if such relationship does not exist in the ground truth tree $(F P)$.

Different lineages accuracy: similarly to the previous measure, we check whether mutations in different branches are correctly inferred or if any pair of mutation is erroneously inferred in different branches.

Figures 10, 11 and 12 show very interesting results. Regarding the results of SCITE and SASC, as expected, we observe a severe drop in performance when used in combination with $k$-means, affinity propagation, hierarchical agglomerative, BIRCH and spectral clustering methods. This fact is supported by the gap in the precision of the methods - a low precision indeed leads to a low accuracy in the tree reconstruction. The trend is still present in the different lineages accuracy, but to a lower extent - this is because, as previously discussed, a cancer inference method can separate clusters of mutations, but when a cluster is computed it is not possible to separate mutations within this cluster.

On the other hand, the results obtained by SCITE when celluloid and $k$-modes are used are much better, in particular, celluloid as a preprocessing step - which leverages our conflict dissimilarity measure - allows SCITE to score higher in both Ancestor-Descendant and Different lineages accuracies than it is able to using unclustered data as input. For this inference tool, using celluloid as a preprocessing step actually helps SCITE to achieve a better score than without it; experiment 3 , being the largest of the three, shows the biggest improvements. SCITE on unclustered data scores an average of 0.7551 and 0.9537 for Ancestor-Descendant and Different lineages respectively, against an average of 0.9358 and 0.9907 after clustering the datasets using celluloid. Moreover, celluloid allows SCITE to achieve a 20x speedup in runtime, on average.

The results obtained by SASC, shown in Figures 10, 11 and 12, are very similar to what is computed by SCITE; in particular celluloid provides much better results than any other clustering method. Once again, on the larger experiment, the gap between the clustering methods is seen the most; in particular, SASC scores an average of 0.9365 and 0.9909 for Ancestor-Descendant and Different lineages respectively, when celluloid is used as a preprocessing step. These results are particularly interesting since SASC is unable to complete in a reasonable amount of time on the unclustered data, hence the reason of this absence from the experimentations.

Unlike the previous tools, SPhyR shows very interesting results since the method itself performs a $k$-means clustering on both mutations and cells given as input. For this reason clustering already clustered data can be a hit-or-miss case, as seen in Figures 10, 11 and 12. In almost all cases, preprocessing the data causes SPhyR to obtain extremely low values in the Ancestor-Descendant measure, while achieving a very good Different lineages score, with the exception of Spectral clustering in all experiments and celluloid with conflict similarity in experiment 1. On the other hand, the Agglomerative clustering seems to have no impact or to slightly improve the tool; this could be a consequence of the high recall and low precision achieved by the clustering algorithm - indeed it is possible that separating well clusters but not merging them very much leads the clustering step of SPhyR to achieve a better result.

\section{Application on real data}

Finally, we run the entire pipeline (clustering + inference method) on an oligodendroglioma IDHmutated tumor [29] consisting of 1842 mutations over 926 cells, to assess if the improvement in the runtimes that we have seen on simulated data carry over to a real dataset. 
Such computation was performed using SCITE, which on unclustered data, takes as long as 68 hours, while adding a preprocessing step with celluloid, we were able to compute the tree within 1 hour, therefore decreasing the time needed by a factor of 70 . Moreover, it is most likely that preprocessing the data with celluloid provides a better phylogeny than SCITE alone, as seen in the experiments on simulated data.

Furthermore we computed the same dataset using SASC, for which such computation was previously not possible with the use of SASC alone, due to the running time required - SASC was not able to provide a solution in a time-frame of three weeks. Figure 13 shows the tree inferred, where each node is one of 100 clusters computed by celluloid.

\section{Discussion}

We have shown how to compare various clustering methods on single cell sequencing data, with three distinct experiments. More precisely, we describe two experiments on synthetic data: the first experiment measures the quality of the clusters computed by the methods, while the second experiment considers the effect of the clustering on the quality of trees obtained downstream from a phylogeny inference tool. Finally, we have described an experiment on real data that measures the usefulness of the clustering procedure, by computing the improvement in the runtime for a large instance.

We have made available at https://github.com/AlgoLab/celluloid/ the entire pipeline that runs the clustering tools on those data, and computes the plots and tables used in the cluster analysis. Our comparison is reproducible and can be easily extended by modifying a Snakemake [19] file.

One of the main conclusions that we can draw from our comparison is that $k$-means is not an adequate choice for clustering single cell data for inferring tumor phylogenies. The second main finding of our paper is that a suitable clustering step, such as celluloid, not only decreases the runtime of the phylogeny inference methods, but can also improve the quality of the inferred phylogenies, as we have shown for SCITE and SASC.

\section{Declarations}

Availability of data and material. Instructions on how to use and replicate all experiments can be found at https://github.com/AlgoLab/celluloid/

Competing interests. The authors declare that they have no competing interests.

Funding. We acknowledge the support of 2018-ATE-0575 and 2017-ATE-0534 grants.

Author's contributions. SC, MDP and GDV devised celluloid and designed the comparison experiments methodology. SC and MDP chose the clustering methods. SC did the experiments. SC, MDP, PB and GDV wrote and revised the manuscript. All authors read and approved the final version of the manuscript.

Acknowledgments. We thank Iman Hajirasouliha and Dana Silverbush for several illuminating discussions. 


\section{References}

[1] M.R. Anderberg. Cluster Analysis for Applications. Academic Press, 1973.

[2] David Brown, Dominiek Smeets, Borbála Székely, et al. Phylogenetic analysis of metastatic progression in breast cancer using somatic mutations and copy number aberrations. Nature Communications, 8:14944, 2017.

[3] F. Chung. Spectral graph theory. In Conference Board of the Mathematical Sciences Regional Conference Series in Mathematics, volume 92.

[4] Simone Ciccolella, Mauricio Soto Gomez, Murray Patterson, Gianluca Della Vedova, Iman Hajirasouliha, and Paola Bonizzoni. Inferring cancer progression from single cell sequencing while allowing loss of mutations. bioRxiv, 268243, 2018.

[5] Simone Ciccolella, Mauricio Soto, Murray Patterson, Gianluca Della Vedova, Iman Hajirasouliha, and Paola Bonizzoni. gpps: An ILP-based approach for inferring cancer progression with mutation losses from single cell data. In IEEE Computational Advances in Bio and medical Sciences, 8th International Conference (ICCABS), 2018. to appear.

[6] Mohammed El-Kebir. SPhyR: tumor phylogeny estimation from single-cell sequencing data under loss and error. Bioinformatics, 34(17):i671-i679, 2018.

[7] Mohammed El-Kebir, Layla Oesper, Hannah Acheson-Field, and Benjamin J. Raphael. Reconstruction of clonal trees and tumor composition from multi-sample sequencing data. Bioinformatics, 31(12):i62-i70, 2015.

[8] E. B. Fowlkes and C. L. Mallows. A method for comparing two hierarchical clusterings. Journal of the American Statistical Association, 78(383):553-569, 1983.

[9] Brendan J. Frey and Delbert Dueck. Clustering by passing messages between data points. Science, 315(5814):972-976, 2007.

[10] Dan Gusfield. Efficient algorithms for inferring evolutionary trees. Networks, 21(1):19-28, 1991.

[11] Iman Hajirasouliha and Benjamin J. Raphael. Reconstructing Mutational History in Multiply Sampled Tumors Using Perfect Phylogeny Mixtures, pages 354-367. Lecture Notes in Computer Science. Springer Nature, 2014.

[12] D.J. Hand. Discrimination and Classification. John Wiley \& Sons, 1981.

[13] Z. Huang. A fast clustering algorithm to cluster very large categorical data sets in data mining. In the SIGMOD Workshop on Research Issues on Data Mining and Knowledge Discovery, pages 1-8, 1997.

[14] Z. Huang. Extensions to the k-modes algorithm for clustering large data sets with categorical values. Data Mining and Knowledge Discovery, 2(3):283-304, 1998.

[15] Lawrence Hubert and Phipps Arabie. Comparing partitions. Journal of Classification, 2(1):193-218, Dec 1985.

[16] Katharina Jahn, Jack Kuipers, and Niko Beerenwinkel. Tree inference for single-cell data. Genome Biology, 17(1):86, 2016. 
[17] Joe H. Ward Jr. Hierarchical grouping to optimize an objective function. Journal of the American Statistical Association, 58(301):236-244, 1963.

[18] W. Klosgen and J.M. Zytkow. Knowledge discovery in databases terminology. In Advances in Knowledge Discovery and Data Mining, pages 573-592. AAAI Press/The MIT Press, 1996.

[19] Johannes Köster and Sven Rahmann. Snakemake - a scalable bioinformatics workflow engine. Bioinformatics, 2012.

[20] Jack Kuipers, Katharina Jahn, Benjamin J. Raphael, and Niko Beerenwinkel. Single-cell sequencing data reveal widespread recurrence and loss of mutational hits in the life histories of tumors. Genome Research, 27:1885-1894, 2017.

[21] S. Malikic, A.W. McPherson, N. Donmez, and S.C. Sahinalp. Clonality inference in multiple tumor samples using phylogeny. Bioinformatics, 31(9):1349-1356, 2015.

[22] J.B. McQueen. Some methods for classification and analysis of multivariate observations. In the 5th Berkely Symposium on Mathematical Statistics and Probability, pages 281-297, 1967.

[23] A. Sorana Morrissy, Livia Garzia, David J. H. Shih, et al. Divergent clonal selection dominates medulloblastoma at recurrence. Nature, 529(7586):351-357, 2015.

[24] Peter C. Nowell. The clonal evolution of tumor cell populations. Science, 194(4260):23-28, 1976.

[25] Andrew Rosenberg and Julia Hirschberg. V-measure: A conditional entropy-based external cluster evaluation measure. In EMNLP-CoNLL, 2007.

[26] Edith M. Ross and Florian Markowetz. Onconem: inferring tumor evolution from single-cell sequencing data. Genome Biology, 17(1):69, 2016.

[27] Jianbo Shi and Jitendra Malik. Normalized cuts and image segmentation. Technical report, 2000 .

[28] F. Strino, F. Parisi, M. Micsinai, and Y. Kluger. TrAp: a tree approach for fingerprinting subclonal tumor composition. Nucleic Acids Research, 41(17):e165, 2013.

[29] Andrew S. Venteicher, Itay Tirosh, Christine Hebert, Keren Yizhak, Cyril Neftel, Mariella G. Filbin, Volker Hovestadt, Leah E. Escalante, McKenzie L. Shaw, Christopher Rodman, Shawn M. Gillespie, Danielle Dionne, Christina C. Luo, Hiranmayi Ravichandran, Ravindra Mylvaganam, Christopher Mount, Maristela L. Onozato, Brian V. Nahed, Hiroaki Wakimoto, William T. Curry, A. John Iafrate, Miguel N. Rivera, Matthew P. Frosch, Todd R. Golub, Priscilla K. Brastianos, Gad Getz, Anoop P. Patel, Michelle Monje, Daniel P. Cahill, Orit Rozenblatt-Rosen, David N. Louis, Bradley E. Bernstein, Aviv Regev, and Mario L. Suvà. Decoupling genetics, lineages, and microenvironment in idh-mutant gliomas by single-cell rnaseq. Science, 355(6332), 2017.

[30] Ulrike von Luxburg. A tutorial on spectral clustering. Statistics and Computing, 17(4), 2007.

[31] Jiguang Wang, Emanuela Cazzato, Erik Ladewig, et al. Clonal evolution of glioblastoma under therapy. Nature Genetics, 48(7):768-776, 2016. 
[32] Hamim Zafar, Anthony Tzen, Nicholas Navin, Ken Chen, and Luay Nakhleh. SiFit: inferring tumor trees from single-cell sequencing data under finite-sites models. Genome Biology, 18(1):178, 2017.

[33] Tian Zhang, Raghu Ramakrishnan, and Miron Livny. Birch: An efficient data clustering method for very large databases. SIGMOD Rec., 25(2):103-114, June 1996. 


\section{Figures}
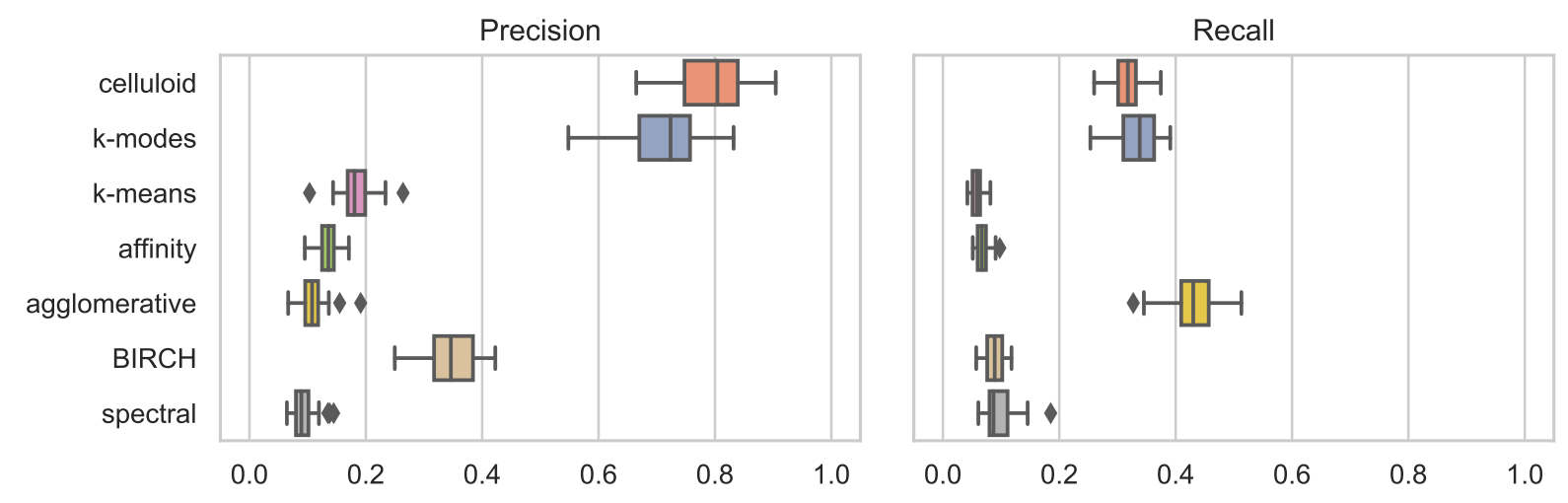

Figure 1: Precision and recall results for experiment 1, generated with a total of 1000 mutations, 100 cells and a clustering size of $k=100$. The plots include results for celluloid, $k$-modes, $k$-means, affinity, agglomerative, BIRCH and spectral clustering.
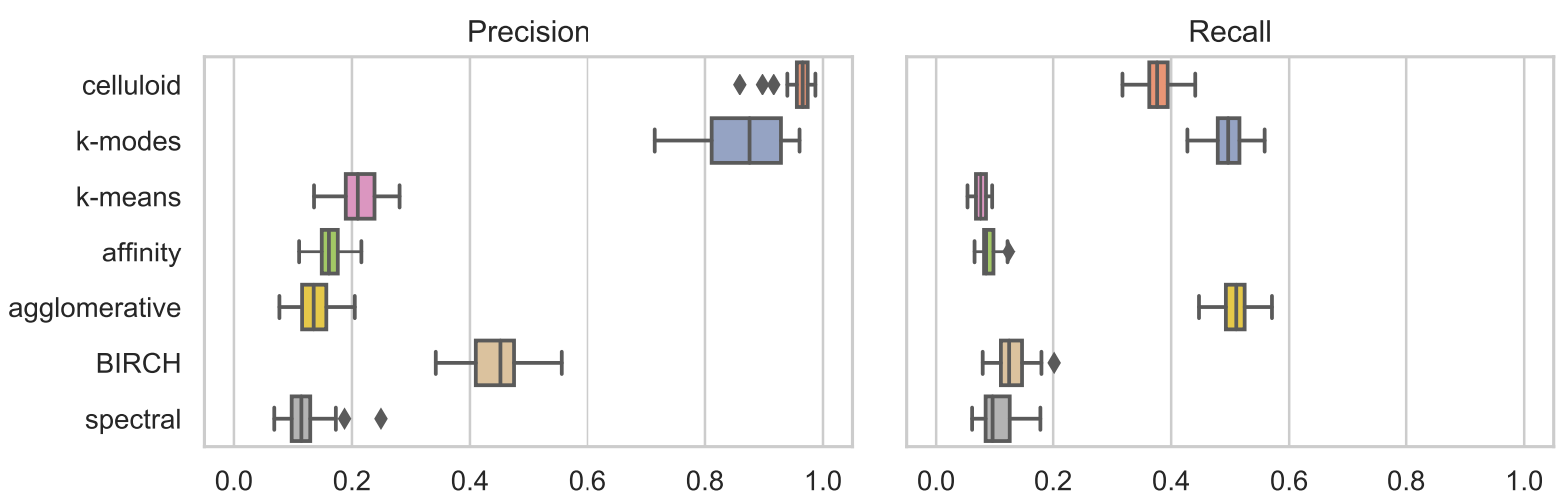

Figure 2: Precision and recall results for experiment 2, generated with a total of 1000 mutations, 200 cells and a clustering size of $k=100$. The plots include results for celluloid, $k$-modes, $k$-means, affinity, agglomerative, BIRCH and spectral clustering. 

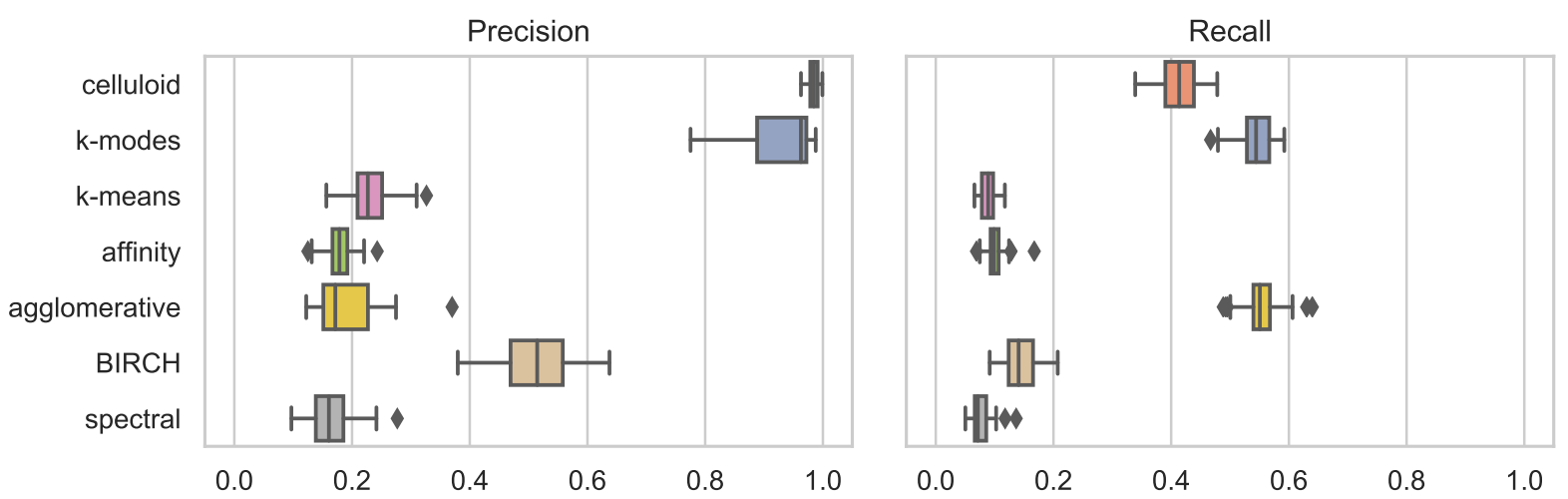

Figure 3: Precision and recall results for experiment 3, generated with a total of 1000 mutations, 300 cells and a clustering size of $k=100$. The plots include results for celluloid, $k$-modes, $k$-means, affinity, agglomerative, BIRCH and spectral clustering.
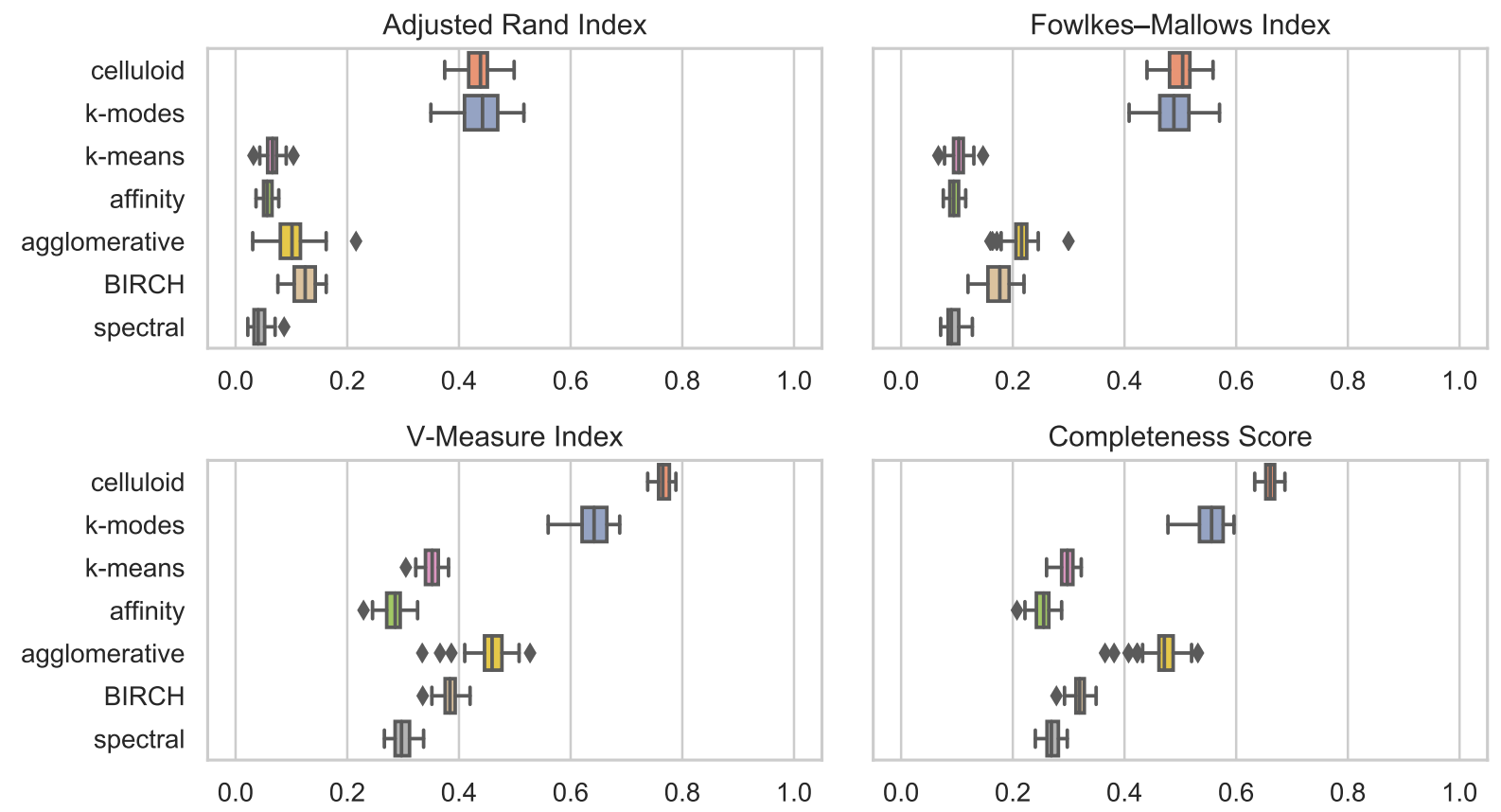

Figure 4: Adjusted Rand index, Fowlkes-Mallows index, Completeness score and V-Measure between all clustering methods and the ground truth for experiment 1, generated with a total of 1000 mutations, 100 cells and a clustering size of $k=100$. The plots include results for celluloid, $k$-modes, $k$-means, affinity, agglomerative, BIRCH and spectral clustering. 

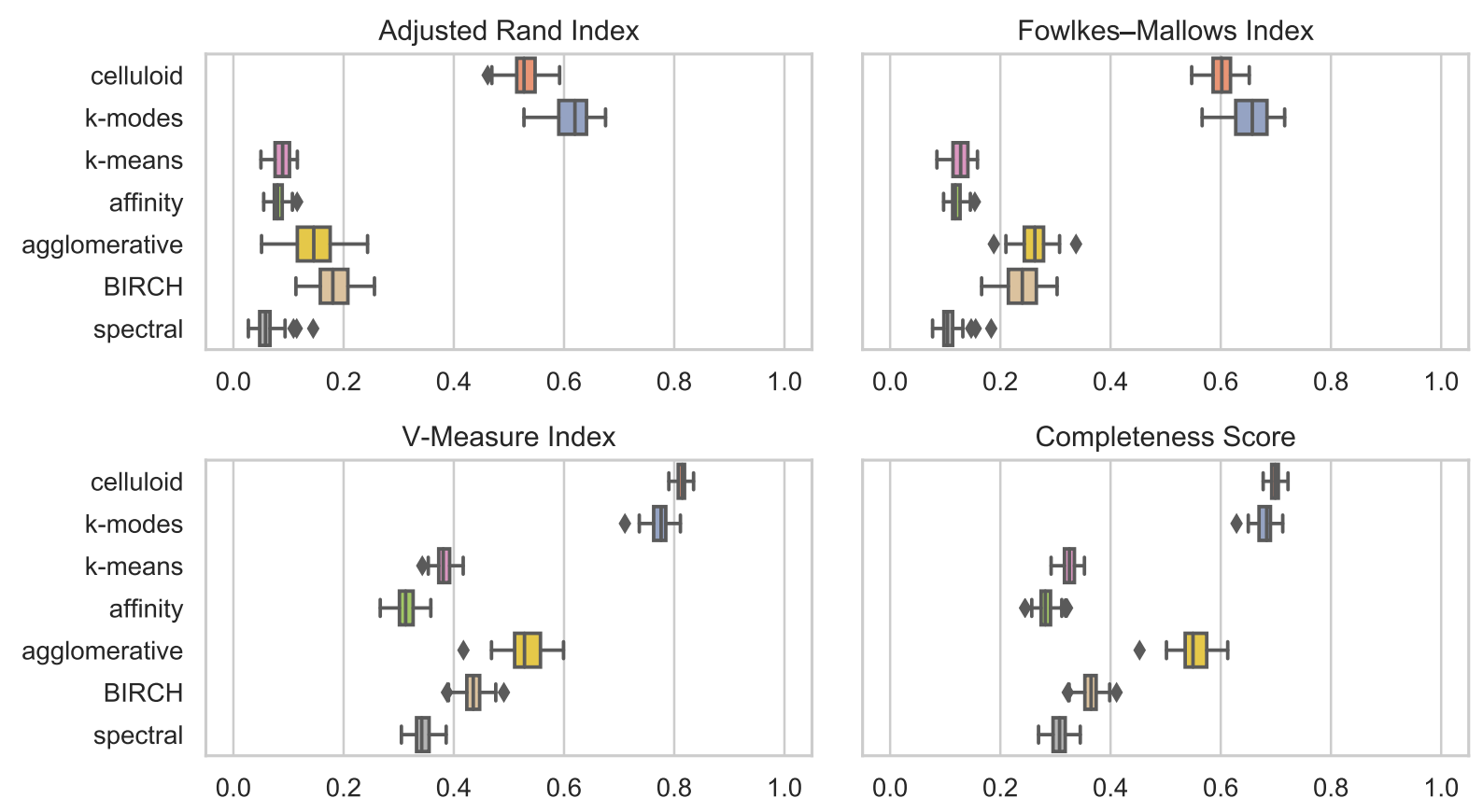

Figure 5: Adjusted Rand index, Fowlkes-Mallows index, Completeness score and V-Measure between all clustering methods and the ground truth for experiment 2, generated with a total of 1000 mutations, 200 cells and a clustering size of $k=100$. The plots include results for celluloid, $k$-modes, $k$-means, affinity, agglomerative, BIRCH and spectral clustering. 

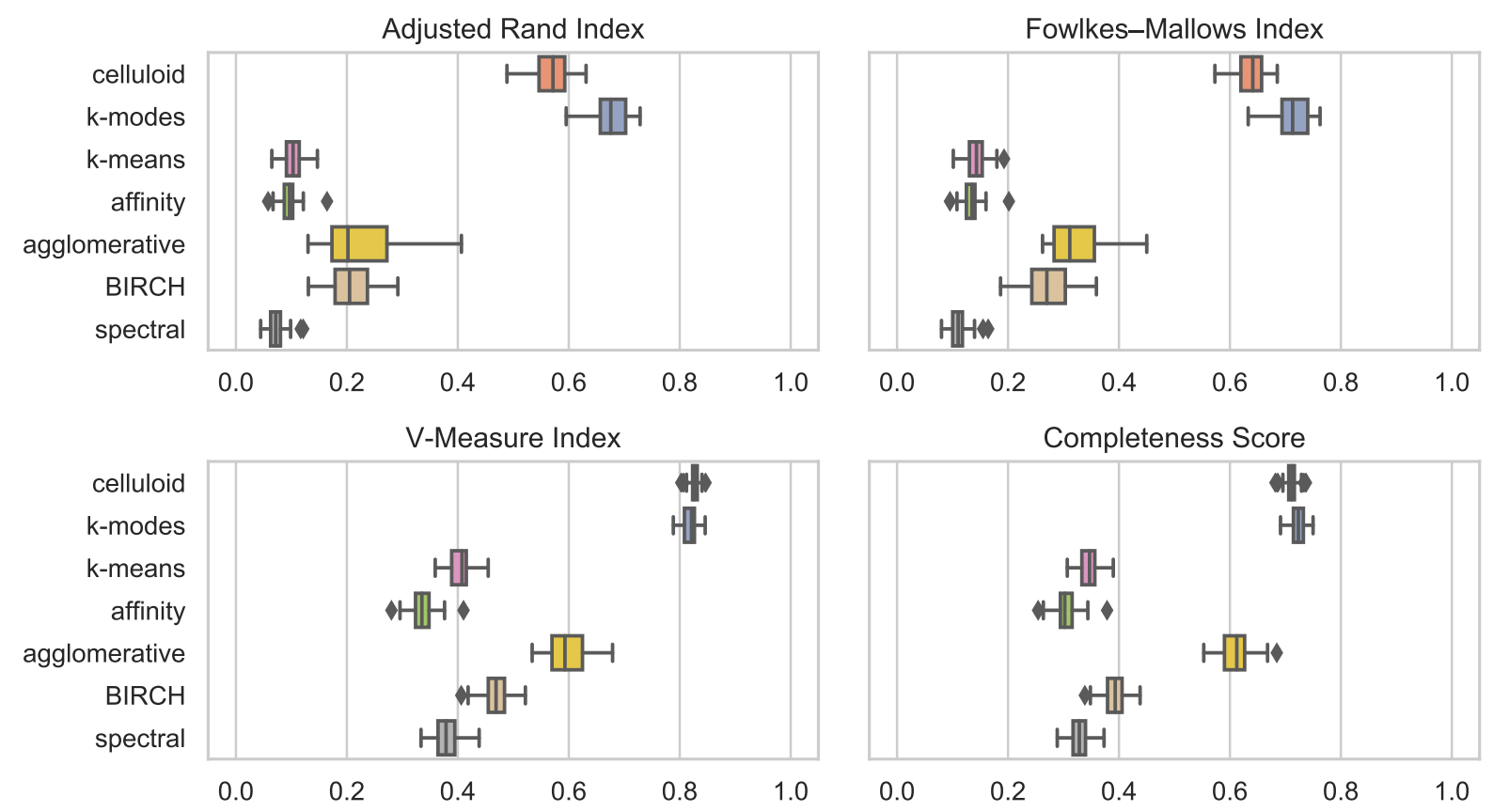

Figure 6: Adjusted Rand index, Fowlkes-Mallows index, Completeness score and V-Measure between all clustering methods and the ground truth for experiment 3, generated with a total of 1000 mutations, 300 cells and a clustering size of $k=100$. The plots include results for celluloid, $k$-modes, $k$-means, affinity, agglomerative, BIRCH and spectral clustering. 


\section{Adjusted Rand Index}

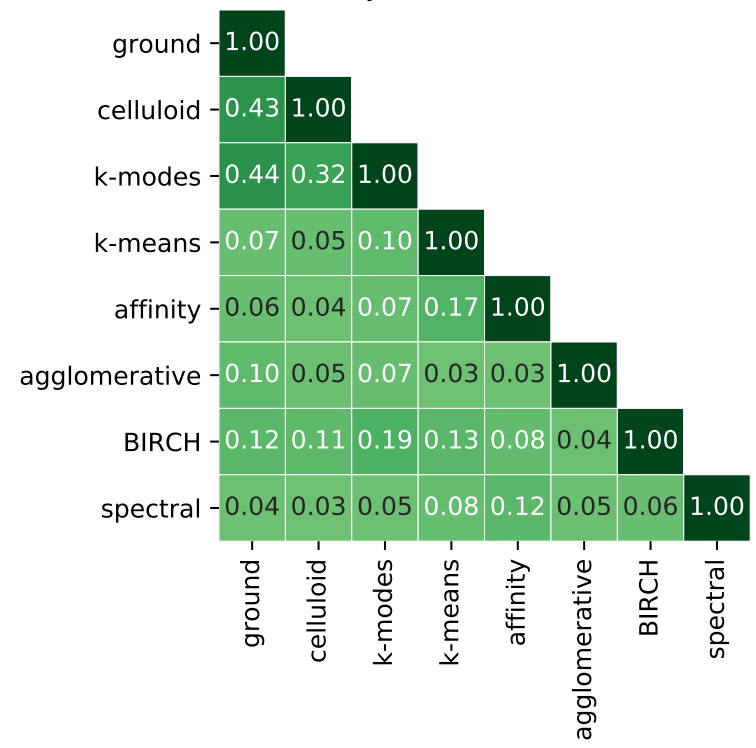

V-Measure Index

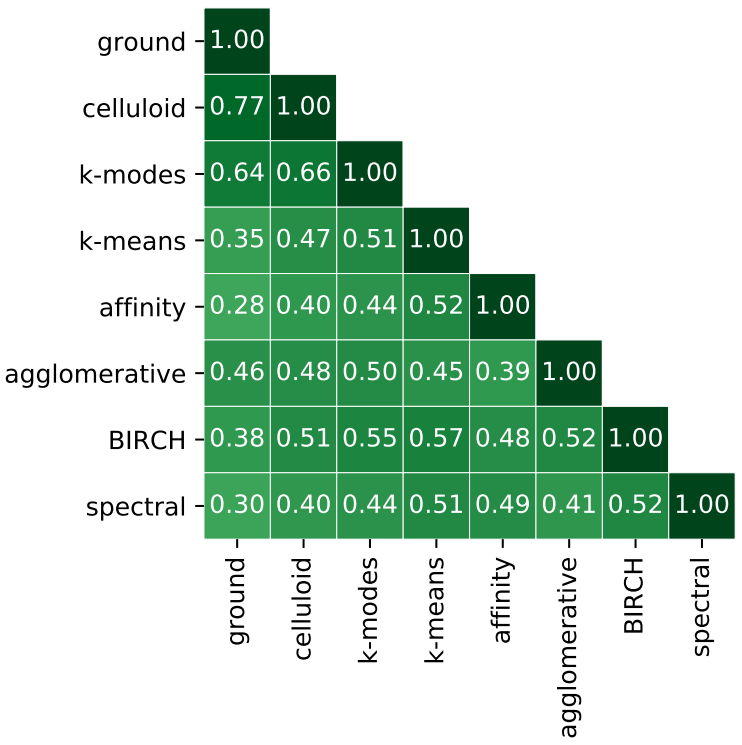

Fowlkes-Mallows Index

1.00

0.501 .00

0.490 .331 .00

$0.100 .07 \quad 0.121 .00$

\begin{tabular}{ll|l|l|l|l}
0.10 & 0.06 & 0.10 & 0.19 & 1.00
\end{tabular}

\begin{tabular}{l|l|l|l|l|l|}
0.21 & 0.15 & 0.17 & 0.11 & 0.12 & 1.00
\end{tabular}

\begin{tabular}{l|l|l|l|l|l|l|l|l|l|l|l}
0.18 & 0.13 & 0.21 & 0.14 & 0.10 & 0.14 & 1.00
\end{tabular}

\begin{tabular}{ll|l|l|l|l|l|l|l}
0.09 & 0.06 & 0.08 & 0.12 & 0.16 & 0.16 & 0.10 & 1.00
\end{tabular}

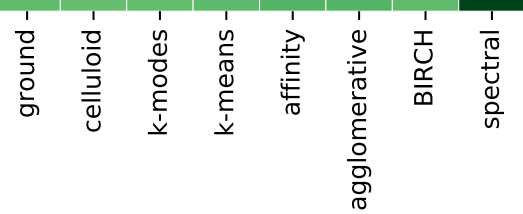

Completeness Score

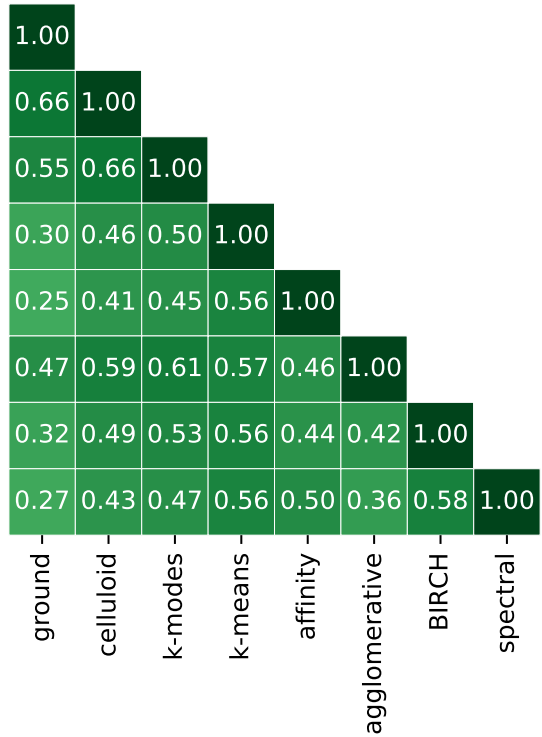

Figure 7: Adjusted Rand index, Fowlkes-Mallows index, Completeness score and V-Measure between all pairs of clustering methods for experiment 1, generated with a total of 1000 mutations, 100 cells and a clustering size of $k=100$. The plots include results for celluloid, $k$-modes, $k$ means, affinity, agglomerative, BIRCH and spectral clustering. Each cell is the average of the scores obtained for each simulated instance. 


\section{Adjusted Rand Index}

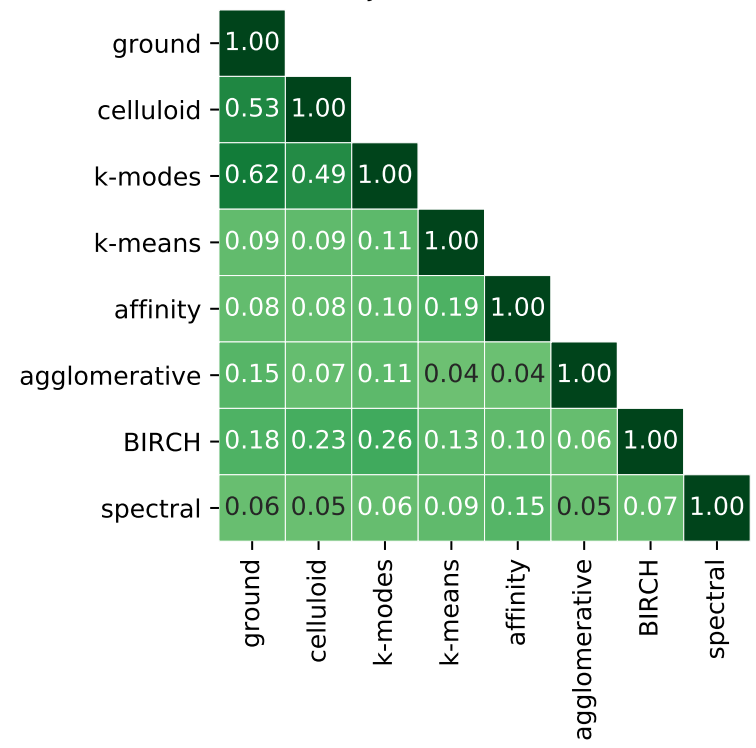

V-Measure Index

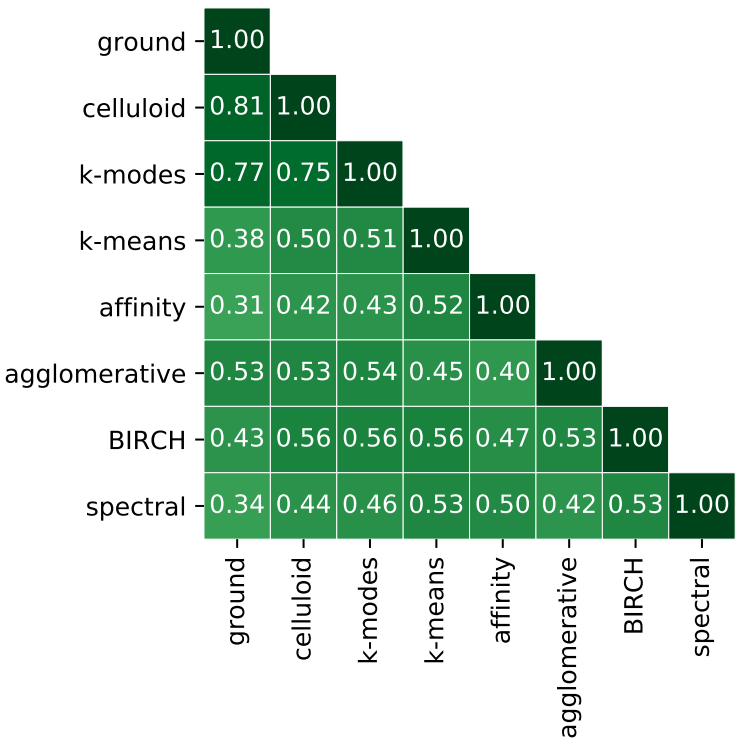

Fowlkes-Mallows Index

1.00

0.601 .00

0.660 .511 .00

0.130 .110 .131 .00

\begin{tabular}{lll|l|l|l|}
0.12 & 0.10 & 0.12 & 0.21 & 1.00
\end{tabular}

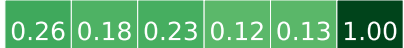

\begin{tabular}{l|l|l|l|l|l|l|}
0.24 & 0.24 & 0.29 & 0.15 & 0.12 & 0.17 & 1.00
\end{tabular}

\begin{tabular}{l|l|l|l|l|l|l|l}
0.11 & 0.08 & 0.10 & 0.13 & 0.19 & 0.16 & 0.10 & 1.00
\end{tabular}

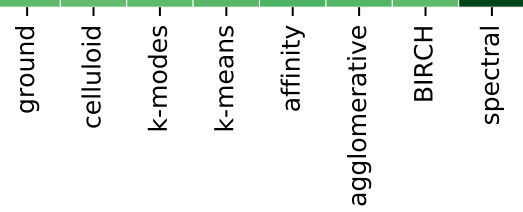

Completeness Score

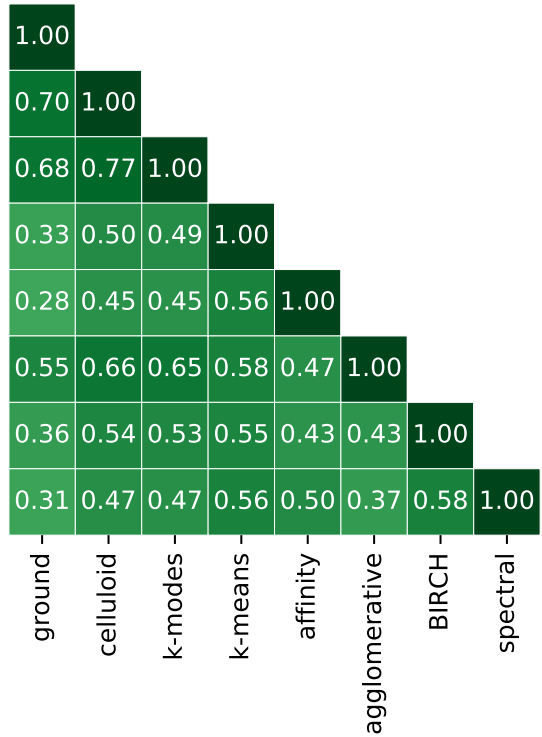

Figure 8: Adjusted Rand index, Fowlkes-Mallows index, Completeness score and V-Measure between all pairs of clustering methods for experiment 2, generated with a total of 1000 mutations, 200 cells and a clustering size of $k=100$. The plots include results for celluloid, $k$-modes, $k$ means, affinity, agglomerative, BIRCH and spectral clustering. Each cell is the average of the scores obtained for each simulated instance. 


\section{Adjusted Rand Index}

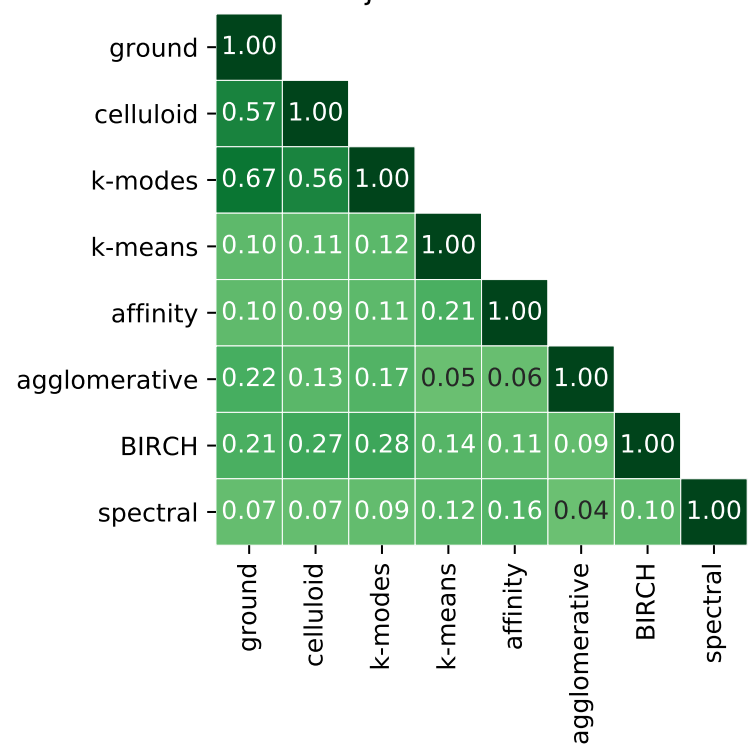

V-Measure Index

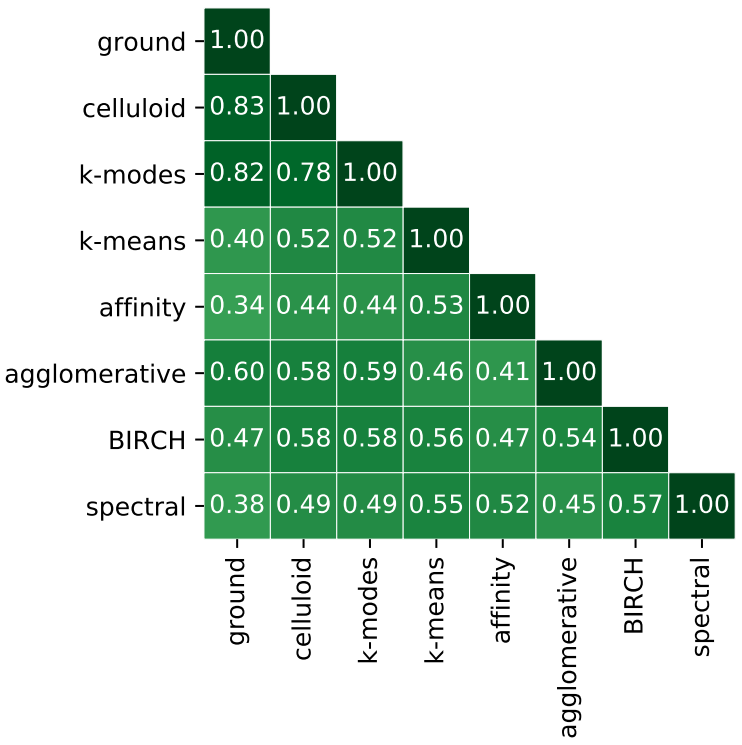

Fowlkes-Mallows Index

1.00

0.641 .00

0.710 .581 .00

\begin{tabular}{l|l|l|l|}
0.14 & 0.13 & 0.15 & 1.00
\end{tabular}

\begin{tabular}{ll|l|l|l|l|l|l|l|l}
0.13 & 0.12 & 0.14 & 0.23 & 1.00
\end{tabular}

\begin{tabular}{l|l|l|l|l|l|}
0.32 & 0.24 & 0.28 & 0.13 & 0.14 & 1.00
\end{tabular}

\begin{tabular}{l|l|l|l|l|l|l|}
0.27 & 0.29 & 0.31 & 0.16 & 0.13 & 0.19 & 1.00
\end{tabular}

\begin{tabular}{l|l|l|l|l|l|l|l}
0.11 & 0.09 & 0.11 & 0.14 & 0.18 & 0.12 & 0.12 & 1.00
\end{tabular}

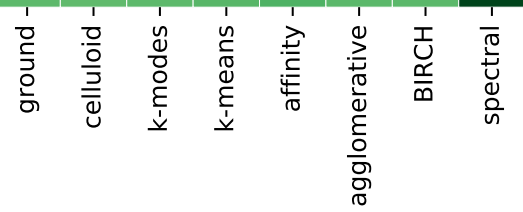

Completeness Score

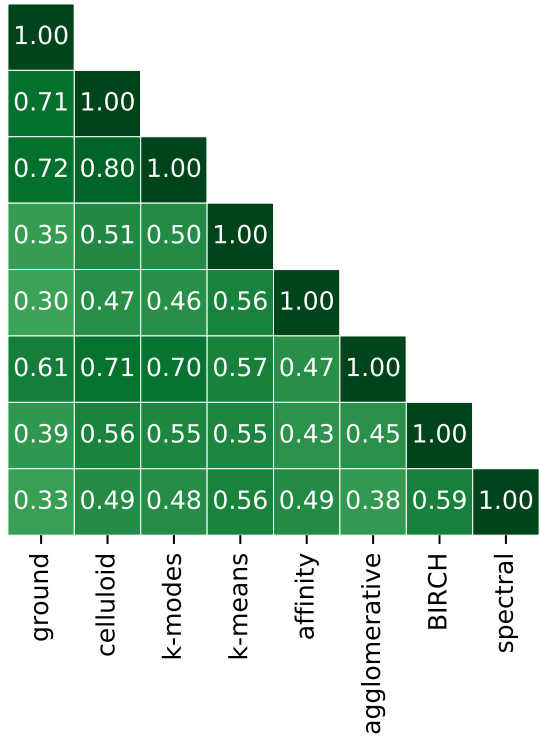

Figure 9: Adjusted Rand index, Fowlkes-Mallows index, Completeness score and V-Measure between all pairs of clustering methods for experiment 3, generated with a total of 1000 mutations, 300 cells and a clustering size of $k=100$. The plots include results for celluloid, $k$-modes, $k$ means, affinity, agglomerative, BIRCH and spectral clustering. Each cell is the average of the scores obtained for each simulated instance. 

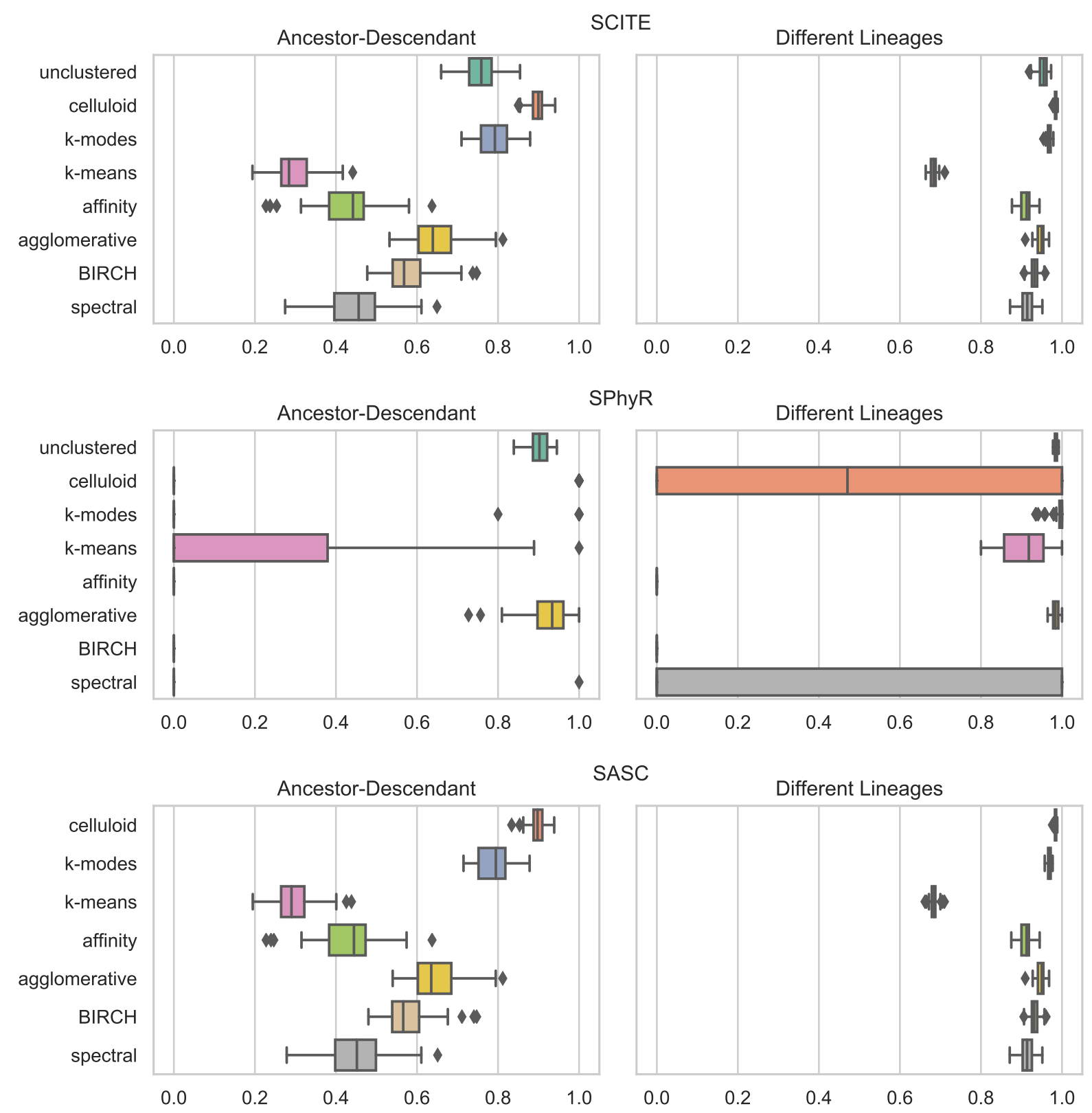

Figure 10: Ancestor-Descendant and Different lineages accuracy measures for experiment 1. Cancer phylogenies are inferred by SCITE (Top), SPhyR (Middle) and SASC (Bottom) using as input both the unclustered data as well as the clusters obtained by celluloid, $k$-modes, $k$-means, Affinity, Agglomerative, BIRCH and Spectral clustering. 

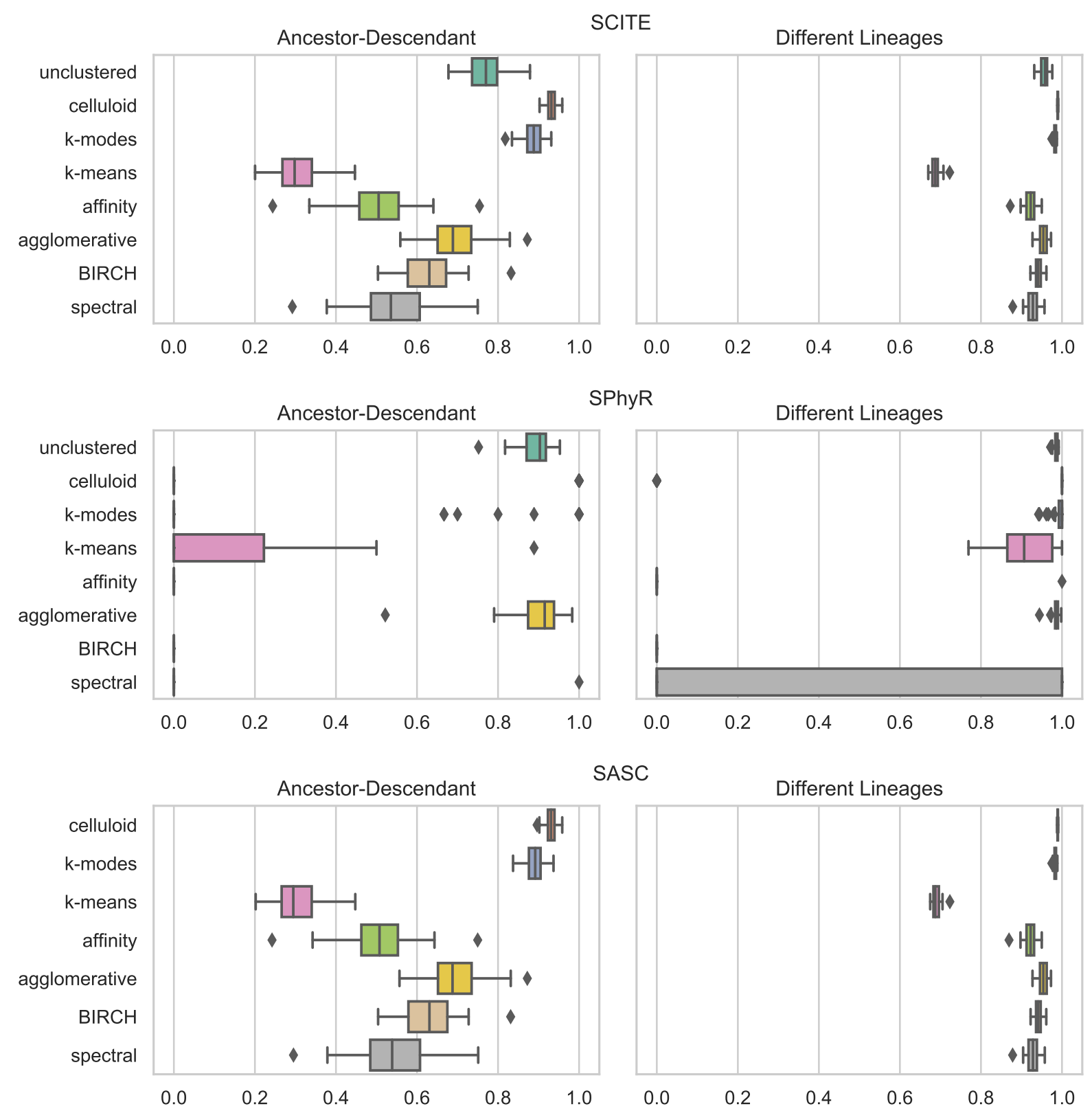

Figure 11: Ancestor-Descendant and Different lineages accuracy measures for experiment 2. Cancer phylogenies are inferred by SCITE (Top), SPhyR (Middle) and SASC (Bottom) using as input both the unclustered data as well as the clusters obtained by celluloid, $k$-modes, $k$-means, Affinity, Agglomerative, BIRCH and Spectral clustering. 

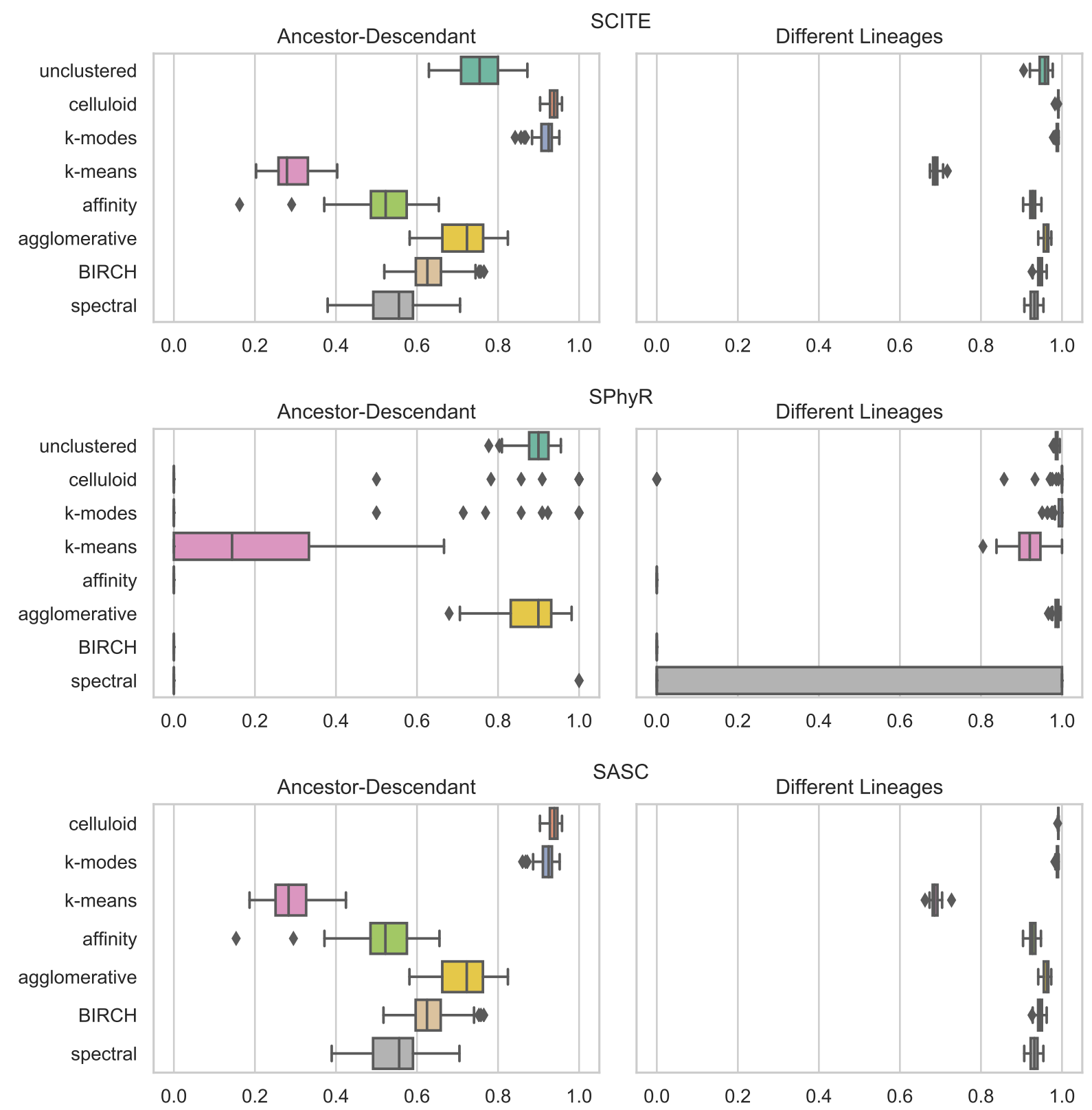

Figure 12: Ancestor-Descendant and Different lineages accuracy measures for experiment 3. Cancer phylogenies are inferred by SCITE (Top), SPhyR (Middle) and SASC (Bottom) using as input both the unclustered data as well as the clusters obtained by celluloid, $k$-modes, $k$-means, Affinity, Agglomerative, BIRCH and Spectral clustering. 


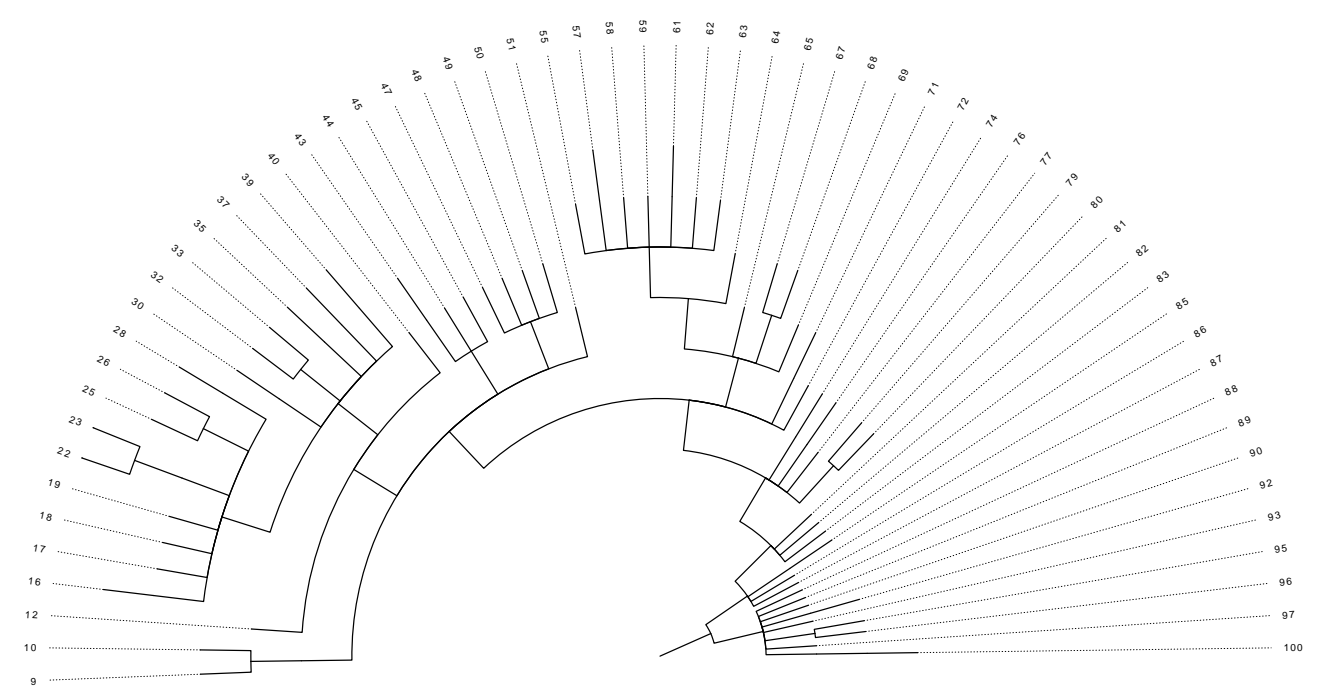

Figure 13: Tree computed on oligodendroglioma IDH-mutated tumor from [29]. The labels on the nodes represent the 100 clusters obtained by celluloid. The tree was inferred using the cancer inference tool SASC. 